\title{
Combined lipiodol marking and video-assisted thoracoscopic surgery in a hybrid operating room
}

\author{
Satoshi Fumimoto ${ }^{1}$, Kiyoshi Sato ${ }^{1}$, Mitsuhiro Koyama ${ }^{2}$, Kazuhiro Yamamoto ${ }^{2}$, Yoshifumi Narumi ${ }^{2}$, \\ Nobuharu Hanaoka ${ }^{1}$, Takahiro Katsumata ${ }^{1}$ \\ ${ }^{1}$ Department of Thoracic and Cardiovascular Surgery, ${ }^{2}$ Department of Radiology, Osaka Medical College, Takatsuki, Osaka, Japan \\ Contributions: (I) Conception and design: S Fumimoto; (II) Administrative support: K Sato; (III) Provision of study materials or patients: N Hanaoka; \\ (IV) Collection and assembly of data: S Fumimoto; (V) Data analysis and interpretation: S Fumimoto; (VI) Manuscript writing: All authors; (VII) \\ Final approval of manuscript: All authors. \\ Correspondence to: Satoshi Fumimoto, MD. Department of Thoracic and Cardiovascular Surgery, Osaka Medical College, 2-7 Daigaku-cho, Takatsuki, \\ Osaka 569-8686, Japan. Email: tho089@osaka-med.ac.jp.
}

Background: The development of diagnostic technology has led to detection of an increasing number of small pulmonary nodules (SPNs), which can be difficult to locate intraoperatively. Here, we report our experience performing single-stage lipiodol localization and surgical resection in a hybrid operating room (OR).

Methods: Between June 2016 and August 2017, 30 patients with 32 SPNs underwent sliding gantrybased multidetector computed tomography (MDCT)-guided lipiodol marking followed by video-assisted thoracoscopic surgery (VATS) in a hybrid OR. After induction of general anesthesia, all nodules were marked with $0.2 \mathrm{~mL}$ lipiodol under MDCT fluoroscopic guidance, followed by immediate VATS.

Results: The mean SPN diameter and distance from the pleural surface were $10.7 \pm 4.5 \mathrm{~mm}$ (range, $5.0-21.0 \mathrm{~mm}$ ) and $18.0 \pm 9.0 \mathrm{~mm}$ (range, 2.8-32.0 $\mathrm{mm}$ ) respectively. The MDCT-guided localization procedure required $15.8 \pm 6.0 \mathrm{~min}$ (range, $8.0-32.0 \mathrm{~min}$ ). All the nodules were marked with lipiodol and detected during fluoroscopy as a clear spot. The median deviation between the radio-opaque nodule and the target nodule was 7.8 $\pm 3.6 \mathrm{~mm}$ (range, 3.0-20.0 mm). In two cases, MDCT scans performed after completion of marking revealed mild pneumothorax, which did not need further intervention. VATS resection was converted to thoracotomy in two patients because of strong pleural adhesions and intraoperative bleeding from the pulmonary vein. No other complications occurred during the combined approach, and there was no intra- or post-operative mortality or morbidity.

Conclusions: These results suggest that a combined approach using MDCT-guided lipiodol marking followed by VATS is feasible and has acceptable accuracy in resection of SPNs.

Keywords: Hybrid operating room (hybrid OR); small pulmonary nodules (SPNs); video-assisted thoracoscopic surgery (VATS); lipiodol marking; sliding gantry

Submitted Mar 29, 2018. Accepted for publication Apr 25, 2018.

doi: $10.21037 /$ jtd.2018.05.28

View this article at: http://dx.doi.org/10.21037/jtd.2018.05.28

\section{Introduction}

Advances in high-resolution computed tomography (CT) imaging and the availability of low-dose CT screening for lung cancer detection in asymptomatic patients are increasing the detection rate of small pulmonary nodules
(SPNs) in the peripheral lung parenchyma $(1,2)$. SPNs or nodules with ground-glass opacity (GGO) are difficult to localize because they are not visible, and manual palpation is sometimes needed during examination of pulmonary lesions. With the current shift toward minimally invasive surgery, video-assisted thoracoscopic surgery (VATS) has become 
Table 1 Clinical and radiological characteristics of the patient in the case series

\begin{tabular}{lc}
\hline Variables & Values \\
\hline Sex, \%male & $46.7(14 / 30)$ \\
Median age, mean $\pm \mathrm{SD}$ (range) (years) & $65.6 \pm 9.2(53.0-78.0)$ \\
Body mass index, & $24.2 \pm 2.4(19.1-28.0)$ \\
mean $\pm \mathrm{SD}\left(\right.$ range) $\left(\mathrm{kg} / \mathrm{m}^{2}\right)$ & \\
Nodule size, mean $\pm \mathrm{SD}(\mathrm{range})(\mathrm{mm})$ & $10.7 \pm 4.5(5.0-21.0)$ \\
Distance between the nodule and & $18.0 \pm 9.0(2.8-32.0)$ \\
pleura, mean $\pm \mathrm{SD}(\mathrm{range})(\mathrm{mm})$ & \\
Nodule morphology $(\mathrm{N})$ & 13 \\
Pure ground glass opacity & 12 \\
Mixed nodule & 7 \\
Solid nodule & \\
Location (N) & 10 \\
Right upper lobe & 2 \\
Middle lobe & 6 \\
Right lower lobe & 6 \\
Left upper lobe & \\
Left lower lobe & \\
\hline
\end{tabular}

common for resection of SPNs because of its minimal postoperative morbidity and mortality, along with less pain and a better quality of life, compared to open thoracotomy $(3,4)$. Successful VATS for resection of SPNs largely relies on the ability to identify lesions intraoperatively by direct visualization or palpation. However, if the target nodule is $<10 \mathrm{~mm}$ in diameter and the distance to the pleura is $>10 \mathrm{~mm}$, the failure rate of lung nodule localization during VATS is $100 \%$, and this is the most common reason for conversion to open thoracotomy (5).

Various methods have been developed to localize SPNs preoperatively and allow accurate resection by VATS. Among these, CT-guided localization is currently the most common (6). In this method, SPNs are initially localized by $\mathrm{CT}$ in a radiology department, followed by surgical resection in an operating room (OR). However, the separate performance of the two procedures can pose substantial risks for pneumothorax, hemothorax, and localizer dislodgement, which are major obstacles to implementation of this approach in surgical practice. These potential risks may be minimized by performing the localization procedure and lung surgery in a hybrid OR equipped with a suitable localization system (7).

Among CT-guided localization techniques, lipiodol marking is accurate and safe and has a high success rate $(6,8-14)$. In addition, unlike other techniques, lipiodol marking permits resection of lesions while verifying the stapled margin in real time (15). Lipiodol marking is the most appropriate method for small pulmonary lesions for several reasons, but postprocedure complications such as pneumothorax and hemorrhage can occur, as with other techniques, most such events can be resolved with conservative management, but further reduction of the risk of these events would be helpful while maintaining the advantages of lipiodol marking. This might be achieved through localization and resection of SPNs using a single-stage procedure in a hybrid OR. The aim of this study was to evaluate the feasibility and safety of this combined approach.

\section{Methods}

\section{Patients and eligibility for the study}

The study was approved by the Institutional Review Board of Osaka Medical College Hospital (approval No. 1905). Written informed consent was obtained from all patients after they received an explanation of the risks and benefits of the procedure. The medical records of 30 patients who underwent a combined approach with lipiodol marking of SPNs using sliding gantry-based multidetector computed tomography (MDCT) immediately followed by resection of SPNs in a hybrid OR from June 2016 to August 2017 were reviewed. Data were collected for SPNs that were thought to be difficult to localize during thoracoscopy. Selection for preoperative localization was based on at least one of the following CT findings: lesion diameter $\leq 10 \mathrm{~mm}$; distance from pleural surface $>5 \mathrm{~mm}$; or a lesion mostly comprising GGO. Clinical and radiologic characteristics of the patients are shown in Table 1. Of the 30 patients, 28 had a single nodule and 2 had two nodules. The patients $(46.7 \%$ male) had a mean age of $65.6 \pm 9.2$ years (range, 53.0-78.0 years) and a mean body mass index (BMI) of $24.2 \pm 2.4 \mathrm{~kg} / \mathrm{m}^{2}$ (range, $19.1-28.0 \mathrm{~kg} / \mathrm{m}^{2}$ ). All the nodules were detected on chest CT, but could not be detected clearly on chest roentgenograms. The mean SPN diameter was $10.7 \pm 4.5 \mathrm{~mm}$ (range, 5.0-21.0 mm) and the mean distance from the pleural surface was $18.0 \pm 9.0 \mathrm{~mm}$ (range, $2.8-32.0 \mathrm{~mm}$ ). Most nodules were pure GGO lesions (13/32) and the most common location was the right 
upper lobe (10/32).

\section{Pre-procedure workup}

The hybrid OR was equipped with a sliding gantrybased MDCT (Somatom Definition AS+SLID, Siemens, Germany) (Figure 1) that could be used near the surgical table or outside the hybrid OR. General anesthesia was performed on the free-floating table and the patient was intubated with a double lumen endotracheal tube. The patient was positioned in a supine or prone position to establish the optimal access route for needle insertion, depending on the location of the pulmonary lesion.

\section{Marking technique}

A radiologist (M Koyama) and a thoracic surgeon (S Fumimoto) with experience in the method planned the

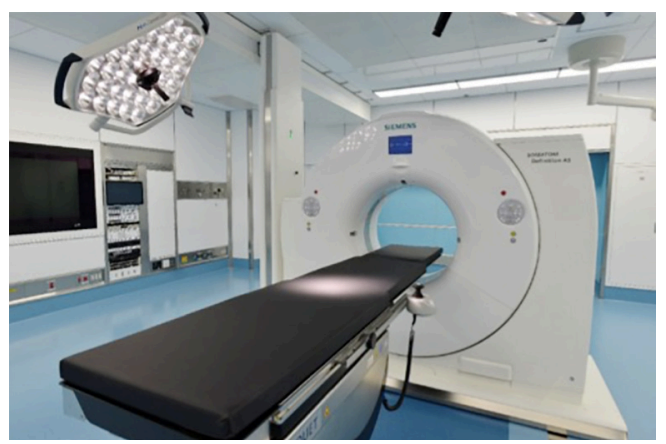

Figure 1 Photograph of the hybrid OR equipped with a sliding gantry-based MDCT system. OR, operating room; MDCT, multidetector computed tomography. needle routes and performed the lipiodol-localization procedure. A scaled paper with metal wires was placed firmly on the patient, and the sliding gantry-based MDCT scan was performed without moving the floating table. The shortest distance from the nodule to the thoracic wall was selected as the injection site. The site for marker injection was marked on the skin, and the angle and depth of the needle required to reach the nodule were determined. A 23 -gauge needle was introduced through the skin to the perilesional pulmonary parenchyma (Figure $2 A$ ). The syringe was withdrawn to confirm that no blood or air had flowed backward, and then $0.2 \mathrm{~mL}$ of lipiodol (Lipiodol UltraFluid; Laboratory Guerbet; Aulnay-Sous-Bois, France) was carefully injected adjacent to the lesion in a single shot. Ventilation was stopped during the marking procedure. The presence of radio-opaque nodules was confirmed by MDCT after completion of marking (Figure $2 B$ ). Subsequently, two-lung ventilation was shifted to one-lung ventilation. The distribution of lipiodol in surrounding pulmonary structures, such as the pulmonary parenchyma, central bronchus, pleural space, and adjacent segments, was examined on the final CT images.

\section{D CT simulation}

After injection of lipiodol, MDCT images were recorded with $2-\mathrm{mm}$ data slices and saved on a computer server. The local area network was connected to a computer with Synapse (Fujifilm Medical Co., Tokyo, Japan), a medical imaging and information management system, at Osaka Medical College Hospital. Vincent (Fujifilm Medical Co.) is an application for imaging analyses using Synapse that can display $2 \mathrm{D}$ multiplanar reconstruction images as virtual
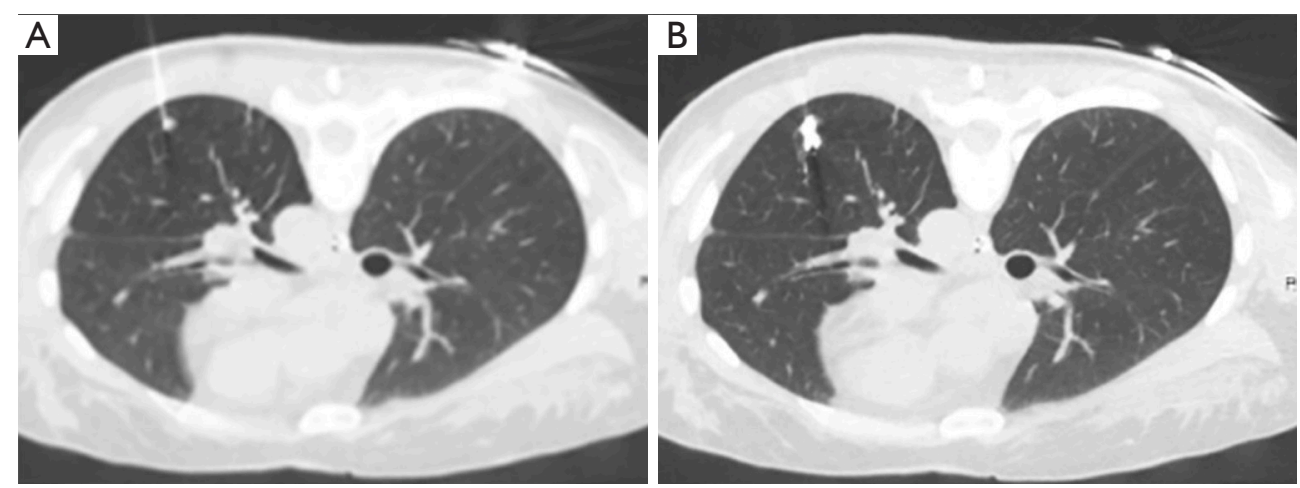

Figure 2 Lung window of the preoperative CT scan in a prone position. (A) A 23-gauge needle located in the perilesional area in the left lower lobe; (B) a volume of $0.2 \mathrm{~mL}$ of lipiodol was injected adjacent to the target lesion. CT, computed tomography 


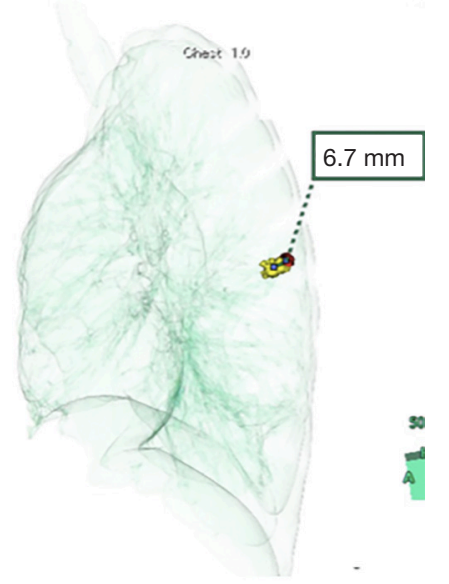

Figure 3 The target lesion (red) and radio-opaque nodule (yellow) with accumulated lipiodol. The deviation was measured as the distance between the centers of the target lesion and nodule on the 3D CT image. CT, computed tomography.

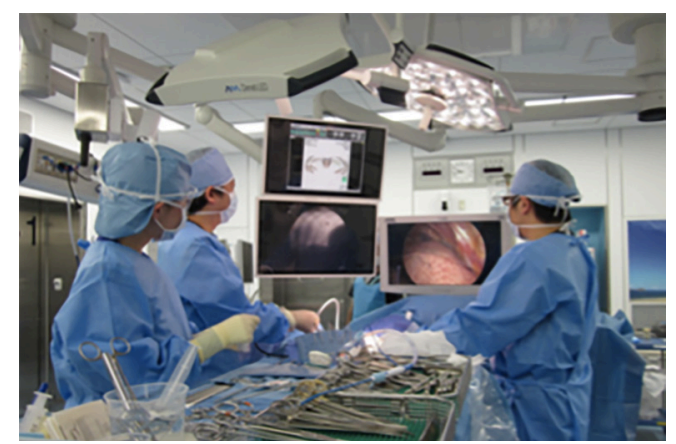

Figure 4 Photograph of the intraoperative scene. The surgeon identifies the target lesion with the thoracoscope monitor and with reference to the fluoroscopic image and the 3D CT image. CT, computed tomography.

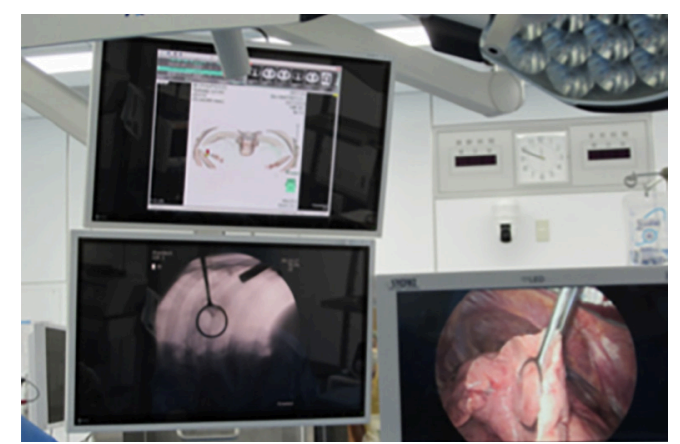

Figure 5 The radio-opaque nodule is grasped with ring-shaped forceps during fluoroscopy. sonography corresponding to the angle in the plane of $3 \mathrm{D}$ volume image data. Reconstruction of the location of the pulmonary lesion and radio-opaque nodule was performed using the $3 \mathrm{D}$ volume-rendering method. The surgeon (S Fumimoto) processed reconstruction of the 3D image at the workstation in the hybrid OR, while moving the patient's position. After completion of reconstruction of the 3D image, the precision of lipiodol marking was evaluated by measuring the deviation from the center of the radio-opaque nodule to the center of the target SPN (Figure 3).

\section{Resection technique}

Immediately after localization was completed, the patient was repositioned into the lateral decubitus position with one-lung ventilation for surgery. The interval between completion of marking and starting of surgical resection was recorded as the repositioning time. Thereafter, a $\mathrm{C}$-arm-shaped fluoroscopy unit was placed horizontally in an appropriate position. A $1-\mathrm{cm}$ incision for the camera port at the seventh intercostal space midaxillary line and a $3-\mathrm{cm}$ incision at the fourth intercostal space anterior axial line were routinely used. Finger palpation to identify SPNs was not conducted in all cases. Based on the fluoroscopy and reconstructed 3D imaging, radio-opaque nodules were detected and the positional relationship between the nodule and the tumor was confirmed (Figure 4). Thereafter, the radio-opaque nodule was grasped with ring-shaped forceps during fluoroscopy (Figure 5) and then resected with an endostapler to maintain the surgical margin under fluoroscopic guidance. Successful resection of the nodule was confirmed by viewing the nodule within the resected specimen during fluoroscopy. Once wedge resection was completed, the resected specimen was sent for frozen section examination after evaluation of the surgical margin. If invasive lung cancer was diagnosed, definitive treatment of lobectomy and lymph node dissection was performed.

\section{Data collection and analysis}

Clinical and pathological records were reviewed retrospectively. Continuous data are expressed as a median with interquartile ranges and analyzed by Mann-Whitney $\mathrm{U}$ test, with $\mathrm{P}<0.05$ defined as significant. All analyses were performed with SPSS (v.20.0; IBM SPSS Inc., Armonk, NY, USA). 
Table 2 Details of the lipiodol marking in the case series

\begin{tabular}{lc}
\hline Variables & \multicolumn{1}{c}{ Values } \\
\hline $\begin{array}{l}\text { Time required for marking, } \\
\text { mean } \pm \text { SD (range) (min) }\end{array}$ & $15.8 \pm 6.0(8.0-32.0)$ \\
Patient's position (N) & 19 \\
Supine & 11 \\
Prone & $7.8 \pm 3.6(3.0-20.0)$ \\
Distance between radio-opaque nodule & \\
and SPN, mean \pm SD (range) (mm) & $6.7 \pm 2.6(3.0-15.0)$ \\
Time required for repositioning, & \\
mean \pm SD (range) (min) & \\
Distribution in surrounding area (N) & 23 \\
None & 5 \\
Lung parenchyma & 4 \\
Peripheral bronchus & 0 \\
Central bronchus & 0 \\
Pleural space & 0 \\
Migration to another segment & \\
\hline
\end{tabular}

SPN, small pulmonary nodule.

\section{Results}

All SPNs were marked on the MDCT image and resected in the hybrid OR. A summary of the lipiodol marking procedure is given in Table 2. The mean total marking time was $15.8 \pm 6.0 \mathrm{~min}$ (range, 8.0-32.0 $\mathrm{min}$ ). Nineteen patients were placed in a supine position, and 11 in a prone position. The median deviation between the radio-opaque nodule and target SPN was $7.8 \pm 3.6 \mathrm{~mm}$ (range, 3.0-20.0 mm). Regarding complications associated with lipiodol marking, mild pneumothorax was found in a MDCT scan performed after completion of marking in two patients. Complications such as air embolism, hemothorax and hemoptysis did not occur. The mean repositioning time (the interval from completion of marking to initiation of surgical resection) was $6.7 \pm 2.6 \mathrm{~min}$ (range, $3.0-15.0 \mathrm{~min}$ ). The radio-opaque nodule was identified without distribution of lipiodol to surrounding lung structures in 23 markings (72\%), and with distribution to pulmonary parenchyma in 5 (16\%) and to peripheral bronchi in $4(13 \%)$. There was no case with distribution to other lung structures. The distributed lipiodol was detected as a dot-like shape in the pulmonary parenchyma and bronchi.

A summary of the surgical procedure and pathology is
Table 3 Details of the surgical procedure and pathology in the case series

\begin{tabular}{lc}
\hline Variables & Values \\
\hline Surgical approach (N) & 21 \\
Wedge resection (VATS) & 9 \\
Lobectomy (VATS) & 1 \\
Wedge resection (open) & 1 \\
Lobectomy (open) & $119.0 \pm 54.0$ \\
Time required for operative procedure, & $(55.0-256.0)$ \\
mean \pm SD (range) (min) & 12 \\
Pathology (N) & 10 \\
Adenocarcinoma in situ & 3 \\
Metastasis & 6 \\
Minimum invasive adenocarcinoma & 1 \\
Invasive adenocarcinoma & \\
Squamous cell carcinoma &
\end{tabular}

VATS, video-assisted thoracoscopic surgery.

shown in Table 3. All 30 patients underwent VATS wedge resection, and a frozen-section examination revealed primary lung cancer in nine patients, who then underwent VATS lobectomy and lymphadenectomy. In two patients, thoracoscopic surgery was converted to open thoracotomy because of strong pleural adhesions and intraoperative bleeding from the pulmonary vein. All nodules were marked with lipiodol and were detected during fluoroscopy as a clear spot. There was no case of open thoracotomy in which a radio-opaque nodule was not clearly visualized by intraoperative fluoroscopy. The time for the surgical procedures ("skin to skin") was $119.0 \pm 54.0 \mathrm{~min}$ (range, 55.0-256.0 min).

In pathologic analysis, 12 of the 32 SPNs were diagnosed as adenocarcinoma in situ (AIS), 10 as a metastatic nodule, 3 as minimum invasive adenocarcinoma (MIA), 6 as invasive adenocarcinoma (AD), and 1 as squamous cell carcinoma (SQ). Lesions were all wedge resected with a distance of $\geq 1 \mathrm{~cm}$ from the stapled line, and resection stumps were all negative histopathologically.

The precision of lipiodol marking was examined by comparing the deviation between the radio-opaque nodule and SPN in the lower lobe and upper/middle lobe (Table 4). Correlation analysis showed no significant correlation between clinical characteristics and this deviation. 
Table 4 Comparison of the precision of lipiodol marking between lower lobes and other lobes

\begin{tabular}{|c|c|c|c|}
\hline Variables & $R U L+M L+L U L(N=17)$ & RLL + LLL (N=13) & $P$ value \\
\hline Nodule size, mean \pm SD (mm) & $10.2 \pm 4.9$ & $11.3 \pm 4.0$ & 0.54 \\
\hline Time required for marking, mean $\pm \mathrm{SD}(\mathrm{min})$ & $16.2 \pm 5.9$ & $15.2 \pm 6.3$ & 0.63 \\
\hline Distance between radio-opaque nodule and SPN, mean \pm SD $(\mathrm{mm})$ & $9.0 \pm 4.6$ & $6.9 \pm 2.2$ & 0.11 \\
\hline
\end{tabular}

SPN, small pulmonary nodule; RUL, right upper lobe; ML, middle lobe; RLL, right upper lobe; LUL, left upper lobe; LLL, left lower lobe.

\section{Discussion}

In this case series, we investigated the feasibility of a combined approach with lipiodol marking and VATS in a hybrid OR equipped with a sliding-gantry based MDCT system. This approach had a high success rate and a low complication rate in performance of single-stage lipiodol marking and thoracoscopic resection, and frozen section analysis show that all SPNs were resected with a safe margin.

A previous meta-analysis of three pulmonary nodule localization methods for VATS (hook-wire, microcoil, and lipiodol) showed that lipiodol localization yielded the highest success rate and microcoil localization had the lowest complication rates (6). The lipiodol marking procedure also has the following advantages: (I) prevention of overresection of normal lung tissue around the nodule because lipiodol-marked nodules appear as clear spots of $<1 \mathrm{~cm}$ in size during fluoroscopy; (II) proper central surgical cut lines can be determined because lipiodol can be injected adjacent to target lesions; (III) unlike the hook-wire and microcoil methods, lipiodol is safe and does not necessarily have to be removed from lung tissue; and (IV) lipiodol does not affect pathological findings (8-14).

There are no reports on complications related to lipiodol marking, but there is a possibility of systemic embolism during the marking procedure because lipiodol is insoluble in water. Complications of pneumothorax, hemosputum and hemopneumothorax arise not because of lipiodol itself, but rather because of the needle placed in the pulmonary parenchyma (8-14). This is a particular problem if scheduled surgery is unexpectedly delayed after nodule marking has been performed, since pneumothorax and hemothorax caused by the marking procedure may progress and become severe during the delay or because of physical separation of the CT room and the OR. This is a common disadvantage that cannot be avoided for all percutaneous CT-guided marking procedures. In addition, patients have both physical discomfort and emotional stress due to use of local anesthesia during the marking procedure. The combined approach with lipiodol marking and surgical resection for SPNs in a hybrid OR may solve these problems. VATS immediately after lipiodol marking without transfer of the patient from the procedure room to the OR is likely to reduce the risks associated with transfer and delays, improve surgical workflow by eliminating the need for coordination between the CT room and OR, and reduce both physical discomfort and emotional stress for the patient.

In conventional lipiodol marking, distribution into the lung parenchyma around the nodule is common. This event does not affect detection on intraoperative fluoroscopy, but distribution into the bronchus and pleural space could cause loss of radio-opaque nodules, with the resultant need for additional marking (13). Moreover, there is possibility that lipiodol may migrate into another segment through the bronchus. Yamagami et al. reported a case of pneumonia occurring 16 days after lipiodol marking because lipiodol migrated into the contralateral lung (16). In the current case series, lipiodol distributed only to pulmonary parenchyma and peripheral bronchi, and all the distributed lipiodol was detected as a dot-like shape. There was no loss of a radio-opaque nodule because of this migration, and all nodules were detectable on intraoperative fluoroscopy as a clear spot. The patients in the hybrid OR were intubated with positive ventilation under general anesthesia, and positive ventilation might also minimize the risk of lipiodol migration into the central bronchus or another segment. Moreover, immediate resection does not give time for lipiodol to migrate, and our results showed a low incidence of distribution to surrounding lung structures compared with a previous report (13).

Preoperative localization in the hybrid OR may also have made the lipiodol marking more accurate than the conventional procedure. To measure the precision of lung nodule marking, we reconstructed the MDCT images to give a $3 \mathrm{D}$ volume image, and measured the deviation between the centers of the radio-opaque and target nodules. 
This is the first report to use a reconstructed $3 \mathrm{D}$ image to evaluate the accuracy of the marking procedure. The mean deviation between the two centers was $7.8 \pm 3.6 \mathrm{~mm}$ (range, 3.0-20.0 mm). During the CT-guided preoperative localization under local anesthesia, the most apparent factor that could affect the accuracy of localization is the respiratory cycle. The shift of the diaphragm during this cycle can shift the location of a lung nodule relative to the thoracic wall, especially for nodules in the lower lobe (17). However, there was no significant correlation between clinical characteristics and the deviation for nodules in the lower or upper/middle lobe, which suggests that ceasing respiration during the marking procedure might minimize the deviation resulting from the effect of the respiratory cycle.

Several limitations of this study should be acknowledged. First, the small number of patients weakens the statistical data. Second, most of the SPNs were single lesions, and there were only two cases with multiple lesions. In a case with multiple lesions, the frequency of puncture of lung parenchyma increases, and the incidence of pneumothorax may change. Third, we used a hybrid OR equipped with a sliding gantry-based MDCT system for the combined approach. To our knowledge, this is the first study that has used this MDCT system for performing single-stage lipiodol marking and removal of SPNs. In fact, most similar studies have used cone-beam CT (CBCT) (18-20).

The CBCT system provides greater working space and offers advanced needle planning under real-time needle guidance using a combination of 3D images and fluoroscopy, and has good angulation and rotation $(21,22)$. These advantages facilitate needle placement and speed up the procedure. However, a collision between the rotating $\mathrm{C}$-arm and surgical table may occur when surgeons are trying to engage both sites in the CBCT field of visualization because the current generation of hybrid OR tables is not designed for thoracic surgery $(20,23)$. This concrete risk is especially evident when patients with a large chest cavity are placed in the lateral decubitus position. Moreover, use of CBCT to perform preoperative marking requires additional training and adjustment of the CBCT system by the operator.

A previous study comparing the performance of CBCTguided and conventional CT-guided transthoracic needle biopsy of lung nodules showed that the accuracy, safety and effective dose were similar for these methods (24). Moreover, it was also concluded that these biopsy systems may be alternatively used to optimize available technological resources. Although the context is different, there may be a similar trend between CBCT and MDCT for preoperative marking procedures. Even in the hybrid OR, MDCT is not significantly technically different from conventional CT-guided localization, and no additional training is required. In addition, the patient positioning is not affected by the size of the chest cavities. Therefore, our combined approach using sliding gantry-based MDCT may be easier to introduce for teams familiar with traditional marking methods, compared with a CBCT-guided marking procedure. Future comparative studies will be needed to establish which type of CT gantry, a closed gantry such as that used in MDCT or an open gantry type for CBCT, is more suitable for single-stage marking and lung resection in a hybrid OR.

\section{Conclusions}

The results of this case series show the feasibility and safety of a combined approach using MDCT-guided lipiodol marking followed by VATS for resection of SPNs in a hybrid OR. This method may minimize the risk of complications of lipiodol marking. Although the marking time and incidence of pneumothorax was acceptable, further efforts are needed to decrease intraoperative radiation exposure.

\section{Acknowledgements}

None.

\section{Footnote}

Conflicts of Interest: The authors have no conflicts of interest to declare.

Ethical Statement: This study was performed after having been approved by the Institutional Review Board of Osaka Medical College Hospital (approval No. 1905). All participants provided an informed consent.

\section{References}

1. Krochmal R, Arias S, Yarmus L, et al. Diagnosis and management of pulmonary nodules. Expert Rev Respir Med 2014;8:677-91.

2. Raad RA, Suh J, Harari S, et al. Nodule characterization: subsolid nodules. Radiol Clin North Am 2014;52:47-67.

3. Bendixen M, Jorgensen OD, Kronborg C, et al. Postoperative pain and quality of life after lobectomy 
via video-assisted thoracoscopic surgery or anterolateral thoracotomy for early stage lung cancer: a randomised controlled trial. Lancet Oncol 2016;17:836-44.

4. Vannucci F, Gonzalez-Rivas D. Is VATS lobectomy standard of care for operable non-small cell lung cancer? Lung Cancer 2016;100:114-9.

5. Suzuki K, Nagai K, Yoshida J, et al. Video-assisted thoracoscopic surgery for small indeterminate pulmonary nodules: indications for preoperative marking. Chest 1999;115:563-8.

6. Park CH, Han K, Hur J, et al. Comparative Effectiveness and Safety of Preoperative Lung Localization for Pulmonary Nodules: A Systematic Review and Metaanalysis. Chest 2017;151:316-28.

7. Ng CSH, Chu CM, Kwok MWT, et al. Hybrid DynaCT scan-guided localization single port lobectomy. Chest 2015;147:e76-8.

8. Nomori H, Horio H, Naruke T, et al. Fluoroscopyassisted thoracoscopic resection of lung nodules marked with lipiodol. Ann Thorac Surg 2002;74:170-3.

9. Watanabe K, Nomori H, Ohtsuka T, et al. Usefulness and complications of computed tomography-guided lipiodol marking for fluoroscopy-assisted thoracoscopic resection of small pulmonary nodules: experience with 174 nodules. J Thorac Cardiovasc Surg 2006;132:320-4.

10. Ikeda K, Nomori H, Mori T, et al. Impalpable pulmonary nodules with ground-glass opacity: success for making pathologic sections with preoperative marking by lipiodol. Chest 2007:131:502-6.

11. Kawanaka K, Nomori H, Mori T, et al. Marking of small pulmonary nodules before thoracoscopic resection: injection of lipiodol under CT-fluoroscopic guidance. Acad Radiol 2009;16:39-45.

12. Kim YD, Jeong YJ, I Hoseok, et al. Localization of pulmonary nodules with lipiodol prior to thoracoscopic surgery. Acta Radiol 2011;52:64-9.

13. Miura H, Yamagami T, Tanaka O, et al. CT findings after lipiodol marking performed before video-assisted thoracoscopic surgery for small pulmonary nodules. Acta Radiol 2016;57:303-10.

14. Mogi A, Yajima T, Tomizawa K, et al. Video-assisted

Cite this article as: Fumimoto $\mathrm{S}$, Sato K, Koyama $\mathrm{M}$, Yamamoto K, Narumi Y, Hanaoka N, Katsumata T. Combined lipiodol marking and video-assisted thoracoscopic surgery in a hybrid operating room. J Thorac Dis 2018;10(5):2940-2947. doi: $10.21037 /$ jtd.2018.05.28 thoracoscopic surgery after preoperative CT-guided lipiodol marking of small or impalpable pulmonary nodules. Ann Thorac Cardiovasc Surg 2015;21:435-9.

15. Lin MW, Tseng YH, Lee YF, et al. Computed tomography-guided patent blue vital dye localization of pulmonary nodules in uniportal thoracoscopy. J Thorac Cardiovasc Surg 2016;152:535-44.e2.

16. Yamagami T, Yoshimatsu R, Miura H, et al. Pneumonia occurring after injection of Lipiodol to localize pulmonary nodules before fluoroscopy-aided thoracoscopic resection. Acta Radiol Short Rep 2014;3:2047981613499754.

17. Seo JM, Lee HY, Kim HK, et al. Factors determining successful computed tomography-guided localization of lung nodules. J Thorac Cardiovasc Surg 2012;143:809-14.

18. Ohtaka K, Takahashi Y, Kaga K, et al. Video-assisted thoracoscopic surgery using mobile computed tomography: new method for locating of small lung nodules. J Cardiothorac Surg 2014;9:110.

19. Gill RR, Zheng Y, Barlow JS, et al. Image-guided video assisted thoracoscopic surgery (iVATS): phase I-II clinical trial. J Surg Oncol 2015;112:18-25.

20. Hsieh MJ, Fang HY, Lin CC, et al. Single-stage localization and removal of small lung nodules through image-guided video-assisted thoracoscopic surgery. Eur J Cardiothorac Surg 2017. [Epub ahead of print].

21. Jin KN, Park CM, Goo JM, et al. Initial experience of percutaneous transthoracic needle biopsy of lung nodules using C-arm cone-beam CT systems. Eur Radiol 2010;20:2108-15.

22. El-Sheik M, Heverhagen JT, Alfke H, et al. Multiplanar reconstructions and three-dimensional imaging (computed rotational osteography) of complex fractures by using a C-arm system: initial results. Radiology 2001;221:843-9.

23. Zhao ZR, Lau RW, Ng CS. Hybrid theatre and alternative localization techniques in conventional and singleport video-assisted thoracoscopic surgery. J Thorac Dis 2016;8:S319-27.

24. Rotolo N, Floridi C, Imperatori A, et al. Comparison of cone-beam CT-guided and CT fluoroscopy-guided transthoracic needle biopsy of lung nodules. Eur Radiol 2016;26:381-9. 